\title{
Long-term outcome of 18 years single-center study of surgical treatment of choledochal cyst patients
}

\author{
Wung Sun HAN, Hongbeom KIM, Jae-Seung KANG, Heeju SON, Youngmin HAN, Wooil KWON, Jin-Young JANG* \\ Department of Surgery and Cancer Research Institute, Seoul National University College of Medicine, Seoul, Korea
}

Introduction: There are not many reports of postoperative long-term malignant risk or postoperative sequelae after surgery on choledochal cyst (CC). The purpose of this study was to report the long-term results of operated CC.

Methods: The patients who underwent surgical treatments for CC between 2003 and 2020 at the Seoul National University Hospital were enrolled. Clinicopathologic factors and pre/postoperative CT or MRI were reviewed.

Results: Of the 153 patients, the female was $85 \%$, and the mean age was 41 years old. The most common symptom was abdominal pain. According to the Todani classification, the most common type was 1c (36.6\%). APBDU was $85.6 \%$ and C-P type, P-C type APBDU were observed at $43.8 \%, 41.8 \%$ respectively. Fourteen patients $(9.2 \%)$ had biliary tract cancer including 8 cholangiocarcinomas and 8 gallbladder cancer. Two patients had both cancers. A comparison of patients with and without malignancy showed that age, operative time, EBL were significantly higher in the malignant group, while the diameter of the cyst is significantly smaller in the malignant group. There was no significant difference in APBDU type and Todani classification type between the two groups. The incidence of long-term complication was $14.3 \%$ and the median time interval was 30 months. The two most common complication was cholangitis or stricture (5.9\%). There were 2 cases (1.3\%) of new cancer near the intrapancreatic remnant bile duct.

Conclusions: The $9.2 \%$ of resected CCs had combined malignancy on biliary tracts. Long-term complications such as cholangitis, anastomosis stricture, and new cancer may occur. Therefore continuous surveillance is required. 